\title{
Exchange Rate Volatility Forecasting Using Higher Order Cumulant Function
}

\author{
Sanja Dudukovic \\ Franklin College-Switzerland, Sorengo, Switzerland
}

\begin{abstract}
This paper provides the optimization of the two existing exchange rate volatility models: the GARCH model and the realized volatility model (RV). It shows that the nature of the GARCH controversy does not seem to focus upon the model structure but rather upon the distribution of the squared returns for which second order moments do not represent a sufficient statistic for the parameter estimation. The evidence is based on the daily and intra daily time series data: EUR/USD, USD/JPY, GBP/USD, and USD/CHF during the period from August 2, 2011 to December 1, 2011, taken from Bloomberg. The empirical evidence supports the perception that the GARCH-based volatility models do not capture stylized facts associated with the squared exchange rate returns. The paper shows that both realized volatility and the GARCH volatility forecasts have a third and fourth cumulant different from zero. Its originality lies in the use of the higher order cumulant function to perform the ARMA parameter estimation, by solving the system of modified Yule-Walker difference equations where the autocorrelation function (ACF) is replaced by the third and/or fourth order cumulant function. It is shown that superior whitening and independence of residuals is achieved if HOC ARMA parameter estimation method is used.
\end{abstract}

Keywords: exchange rate volatility, GARCH model, ARMA parameter estimation, higher order cumulant function, non-Gaussian time series, Yule-Walker difference equations

\section{Introduction}

The exchange rate volatility model assumes new importance in the framework of international trade due to two main reasons. Firstly, the introduction of the first Basel Accord, which sets minimum capital reserve requirements to be held by financial institutions proportional to their estimated risks, has further highlighted the significance of volatility. Secondly, volatility forecasts of a stock price are fundamental inputs for pricing derivatives as well as trading and hedging strategies. A basic mean variance analysis also requires estimates of the variance for the assets under consideration. Given these facts, the quest for precise forecasts appears to be still ongoing.

Today there is no doubt that the theoretical model of classical economics, based on the efficient market hypothesis, cannot be confirmed by actual market data (Mandelbrot, 1963). This finding is confirmed by generations of researchers. Surprisingly, as it may seem, this result fails to immanently inspire a determined effort to develop a better theory.

The availability of the high-frequency intraday data has made a modest impact on the modeling of daily

Sanja Dudukovic, Ph.D., Full Professor, Department of International Management, Franklin College-Switzerland.

Correspondence concerning this article should be addressed to Sanja Dudukovic, Franklin College, Department of International Management, Via Ponte Tresa 29, 6924, Sorengo, Switzerland. E-mail: Sdudukovic@fc.edu. 
return volatility. It has become evident that standard volatility models used for forecasting at the daily level cannot readily accommodate the information in intraday data, and generally fail to capture the longer intraday volatility movements sufficiently well. As a result, making forecasts of daily volatility from daily return relying mostly on GARCH (Generalized Autoregressive Conditional Heteroskedasticity) volatility modeling remains a standard practice (Bollerslev, 1982).

Indeed, there are many variants of $\mathrm{ARCH} / \mathrm{GARCH}$ models which are developed to improve the out-of-sample volatility forecasting performance. These models have many strong proponents, who believe that these models are currently the best obtainable forecast estimators. However, most of the empirical studies on the subject in recent years have found no clear-cut results in improving forecasting performances of this class of GARCH models (Poon \& Granger, 2003; Carrol \& Kearvey, 2009). There are studies which confirm a very low coefficient of determination produced by GARCH models. For instance, Andersen et al. (2003) show theoretically that $R^{2}$ for a $\operatorname{GARCH}(1,1)$ model tends to $1 / K$, where $K$ stands for the kurtosis of the distribution of stock returns. This means that the highest $R^{2}$ for Gaussian returns achievable by GARCH models is bounded from above by $1 / 3$. For the exchange rate returns, which have a non-Gaussian distribution, the kurtosis is usually much higher than three, which means that a volatility forecast performance is expected to be worse in practice.

An examination of the relative performance of GARCH models versus simple rules in forecasting volatility is done by Silvey (2007). Although numerous studies have compared the forecasting abilities of historical variance and GARCH models, no clear winner has emerged. In a thorough review of 93 such studies, Poon and Granger (2003) report that only 22 of the studies conclude that historical volatility forecasts out-of-sample future volatility more accurately, while 17 studies find that GARCH models are more accurate. The Dow Jones Industrial Average (DJIA) composite daily data is used to test in- and out-of-sample forecasts, obtained by using GARCH, EGARCH, GRJ, and HS (historical volatility) models (Brooks, 1997). The $R^{2}$ achieved is around $25 \%$ for each of the models.

The theoretical and empirical properties of realized volatility are derived in Andersen, Bollerslev, Diebold, and Labys's (2003) foreign exchange data. Further empirical evidence is provided in Andreou, Pittis, and Spanos's (2001) U.S. equities. Related research into the econometric properties of realized volatility includes Barndorff-Nielsen and Shephard (2002). The heterogeneous autoregressive model of realized volatility (HAR-RV), proposed by Corsi (2009) is shown to be very successful in outperforming several models, such as GARCH, AR, and ARFIMA models.

This paper shows that the nature of this GARCH controversy does not seem to focus upon the model structure but rather upon the distribution of the squared returns for which second order moments do not represent a sufficient statistic for the parameter estimation. The empirical evidence supports the perception that the GARCH-based volatility models do not capture stylized facts associated with the squared exchange rate returns. The evidence is based on the daily and intra daily time series data: EUR/USD, USD/JPY, GBP/USD, and USD/CHF during the period from August 2, 2011 to December 1, 2011, taken from Bloomberg. The paper shows that both realized volatility and the GARCH volatility forecast have a third and fourth cumulants different from zero. Its originality lies in the use of the higher order cumulant function to perform the ARMA parameter estimation and further whiten the forecasting errors, which ultimately have much lower third and fourth order cumulants for 20-50 time lags. The paper also demonstrates the enhanced performance of the realized volatility forecasts, relative to $\mathrm{GARCH}$ volatility forecasts, which comes from the use of high 
frequency returns.

The organization of the paper is as follows: the second section briefly summarizes the literature findings and introduces the problem; the third section presents the GED/GARCH model results as well as RV model results and respective coefficients of determination; the fourth section compares cumulant functions of the volatility forecasting errors based on the HOC-GARCH model with ones obtained using the RV model and suggests the ARMA parameter estimation based on the higher order cumulant function. Ultimately, the last section provides a summary of the results and suggests further research directions.

\section{The Problem and the Model}

The statistical properties of exchange rate returns, common across a wide range of developed stock markets and time periods, are called stylized facts. The stylized statistical properties of asset returns of developed markets are analyzed empirically and subsequently summarized by Cont (2001). They include the following findings: The autocorrelations of asset returns are often insignificant, except for high frequency data ( $\simeq 30$ minutes or less); Heavy tails, with a finite tail index, which is higher than two and lower than five for most data sets studied; Gain/loss asymmetry: one observes large drawdown in stock prices and stock index values but not equally large upward movements; Aggregational gaussianity according to the Central Limit Theorem; Volatility clustering, namely, different measures of volatility display a positive autocorrelation over several days, which quantifies the fact that high-volatility events tend to cluster in time; Conditional heavy tails even after correcting the returns for the volatility clustering; Slow decay of autocorrelation in absolute or squared returns, i.e., non-stationarity; Leverage effect as most measures of the volatility of an asset are negatively correlated with the returns of that asset; Volume/volatility correlation, i.e., the trading volume is correlated with all measures of volatility.

The facts that exchange rate returns are often characterized by volatility clustering - which means that periods of a high volatility are followed by periods of a high volatility and periods of a low volatility are followed by periods of a low volatility - implies that the past volatility could be used as a predictor of the volatility in the next period. As an indication of volatility clustering, squared returns often have significant autocorrelations and consequently can be modeled by using the well-known GARCH model.

Let $e_{t}$ denote a discrete time stationary stochastic process. The $\operatorname{GARCH}(p, q)$ process is given by the following set of equations (Bollerslev, 1982, pp. 42-56):

$$
\begin{gathered}
r_{t}=\log \left(P_{t}\right)-\log \left(P_{\mathrm{t}-1}\right) \\
r_{t}=x\left({ }_{k}\right) g\left({ }_{k}\right)+e_{t} \\
e_{t}=v_{t} \sqrt{ } h_{t} \\
e_{t} / t-1 \\
\approx N\left(0, h_{t}\right) \\
h_{t}=\alpha_{0}+\sum_{1}^{p} \alpha_{i} e_{t-1}^{2}+\sum_{1}^{q} \beta_{j} h_{t-j}
\end{gathered}
$$

where $p_{t}$ represents stock prices; $e_{t}$ represents random returns; $x\left({ }_{k}\right)$ is a vector of explanatory variables; $g\left({ }_{k}\right)$ is a vector of multiple regression parameters; $h_{t}$ is the conditional volatility; $\alpha_{i}$ is autoregressive; and $\beta_{j}$ is the moving average parameter as related to the squared stock market index residuals.

An equivalent ARMA representation of the $\operatorname{GARCH}(p, q)$ model is given by:

$$
e_{t}^{2}=\alpha_{0}+\sum_{1}^{p}\left(\alpha_{i}+\beta_{i}\right) e_{t-\mathrm{i}}^{2}+v_{t}-\sum_{1}^{p} \beta_{j} v_{t-\mathrm{j}}
$$

where $v_{\mathrm{t}}=e_{t}^{2}-h_{t}$ and by definition, it has the characteristics of (i.i.d) white noise. $h_{t}$ is known as GARCH 
volatility. In other words, the $\operatorname{GARCH}(p, q)$ volatility model is an Autoregressive Moving Average (ARMA) model in $e_{t}^{2}$ driven by white noise $v_{t}$. However, the validity of any statistical inference of such model depends on the underlying distribution of the returns.

Most of the studies which use these ARCH and GARCH models document the existence of severe excess skewness and kurtosis in the residuals estimated from their equations. Many researchers attempt to identify the possible causes of and correct or account for the non-normality exhibited by the residuals estimated from GARCH models. In general, the literature attributes such non-normality to model misspecifications and outliers. Despite making theoretical and empirical advances, many of these studies still experience excess kurtosis in the GARCH residuals.

In the case of exchange rate returns, this article argues that the second order moment of the associated probability density distribution is not a sufficient statistic for the ARMA parameter estimation. In fact, it is well known that for a non-Gaussian process, higher order moments exist and are different from zero. Namely, the hypothesis in this article is that higher order cumulants of GARCH forecasting errors contain the information necessary to capture heavy tails, volatility clustering, and long term dependence. More generally, the hypothesis which tested that the stylized facts can be captured by using the higher order cumulant function for the model parameter estimation.

\section{Empirical Analysis}

\section{GARCH Model—Daily Data}

The evaluation and comparison of volatility models are made difficult by the fact that the conditional variance is unobservable. The first approach to getting round this problem, as explained above, is to substitute squared returns for the unobserved conditional variance.

In this study the GED-GARCH model is used for exchange rate volatility. The empirical analysis is based on daily quotations of opening daily exchange rates for the following currency pairs: EUR/USD, USD/JPY, GBP/USD, and USD/CHF during the period from August 2, 2011 to December 1, 2011, taken from Bloomberg and from the OANDA online databases. The common sample of exchange rate descriptors is presented in Table 1.

Table 1

Sample Description for Squared Returns on Daily Exchange Rates

\begin{tabular}{|c|c|c|c|c|}
\hline & $R^{2} \mathrm{EUR} / \mathrm{USD}$ & $R^{2} \mathrm{EUR} / \mathrm{JPY}$ & $R^{2} \mathrm{USD} / \mathrm{CHF}$ & $R^{2} \mathrm{GBP} / \mathrm{USD}$ \\
\hline Mean & 0.0510 & 0.0614 & 0.1724 & 0.0235 \\
\hline Median & 0.0148 & 0.0163 & 0.0280 & 0.0106 \\
\hline Maximum & 0.6462 & 0.8801 & 3.8756 & 0.4532 \\
\hline Minimum & 0.0000 & 0.0000 & 0.0000 & 0.0000 \\
\hline Std. Dev. & 0.0904 & 0.1184 & 0.5174 & 0.0462 \\
\hline Skewness & 3.6131 & 4.1007 & 5.4974 & 6.8811 \\
\hline Kurtosis & 19.9532 & 24.6778 & 35.2496 & 63.2465 \\
\hline Jarque-Bera & $1,712.2990$ & $2,708.3320$ & $5,852.9730$ & $19,254.3400$ \\
\hline
\end{tabular}

Table 1 shows that all the variables are non-Gaussian (according to the skewness, kurtosis, and the Jarque-Bera test for normality). The best significant models found for the squared returns using E-views for 
GED distribution, together with the respective coefficients of determination, are presented in Table 2.

Table 2

GARCH-ARMA Parameter Estimates

\begin{tabular}{|c|c|c|c|c|c|c|c|c|c|}
\hline Parmeters & & $\alpha 1$ & $\alpha 2$ & $\alpha 3$ & $\beta 1$ & $\beta 2$ & $\beta 3$ & $\beta 4$ & $R^{2}$ \\
\hline \multirow[t]{2}{*}{$R^{2} \mathrm{EUR} / \mathrm{USD}$} & Coeff & -0.445 & -0.818 & -0.709 & 0.613 & 0.797 & 0.843 & & 0.144 \\
\hline & St. error & 0.181 & 0.049 & 0.178 & 0.135 & 0.033 & 0.146 & & \\
\hline \multirow[t]{2}{*}{$R^{2} \mathrm{EUR} / \mathrm{JPY}$} & Coeff & & 0.457 & & 0.228 & -0.885 & 0.165 & 0.311 & 0.349 \\
\hline & St. error & & 0.086 & & 0.085 & 0.095 & 0.085 & 0.064 & \\
\hline \multirow[t]{2}{*}{$R^{2} \mathrm{USD} / \mathrm{CHF}$} & Coeff & -0.333 & 0.258 & & 0.927 & & & & 0.274 \\
\hline & St. error & 0.112 & 0.105 & & 0.057 & & & & \\
\hline \multirow[t]{2}{*}{$R^{2} \mathrm{GBP} / \mathrm{USD}$} & Coeff & -0.087 & 0.840 & & & -0.981 & & & 0.067 \\
\hline & St. error & 0.050 & 0.055 & & & 0.023 & & & \\
\hline
\end{tabular}

It can be seen from the Table 2 that the maximum coefficient of determination achieved is $34 \%$. This confirms a low explanatory power of the GARCH model in the case of daily exchange rate volatility. More importantly, the GARCH forecasting errors show persistent higher order central moments. This type of findings is summarized in Table 3.

Table 3

Sample Description for GARCH Volatility Foresting Errors-Residuals

\begin{tabular}{lcccc}
\hline & RES $R^{2}$ EUR/USD & RES $R^{2}$ EUR/JPY & RES $R^{2}$ USD/CHF & RES $R^{2}$ GBP/USD \\
\hline Mean & 0.0020 & 0.0035 & -0.0001 & -0.0012 \\
Median & -0.0063 & -0.0247 & -0.0604 & -0.0095 \\
Maximum & 0.5246 & 0.5782 & 3.8621 & 0.4002 \\
Minimum & -0.1175 & -0.1322 & -0.8913 & -0.0484 \\
Std. Dev. & 0.0866 & 0.0960 & 0.4443 & 0.0449 \\
Skewness & 2.6552 & 2.4278 & 6.0506 & 6.1856 \\
Kurtosis & 14.6592 & 12.8590 & 51.0660 & 55.0894 \\
Jarque-Bera & 813.8517 & 598.8521 & $12,181.5500$ & $14,212.3300$ \\
\hline
\end{tabular}

\section{Realised Volatility and High Frequency Data}

Realised volatility, defined as the sum of intraday, $30 \mathrm{~min}$ squared returns, provides a more accurate estimate of the latent process that defines volatility than is given by daily squared returns.

$$
R V_{t}=\sum_{1}^{48} r_{t, i}^{2}
$$

The theoretical and empirical properties of realized volatility are derived in Andersen, Bollerslev, Diebold, and Labys (2001) for foreign exchange. They find that realised volatility distribution is nearly Gaussian. Further empirical evidence is provided in Andersen, Bollerslev, Diebold, and Ebens (2001) for U.S. equities. A statistical description of the realised volatility is presented in Table 4, while ARMA parameters re-presented in Table 5 .

The results in Table 5 show extremely high coefficient determination for all exchange rates. However, it is to be stressed out that the roots of the characteristic equations are close to the init cycle. That indicates nearly non stationary realised volatilities.

In addition to that problem, there is a problem regarding "calling upon the Central Limit Theorem to justify the use of normal distribution from intraday data because the summation involves only a finite number 
of terms which does not go to infinity" (Andreou, Pittis, \& Spanos, 2001). Further, it is also concluded that averaging is inappropriate because it creates spurious autocorrelation. Therefore, the obtained coefficient of determination must be taken with caution. Incredibly as it may seem, this problem is underestimated in Andersen, Bollerslev, Diebold, and Labys (2003).

Nevertheless, it is interesting to look at the properties of RV forecasting errors. Those errors are neither independent nor identically distributed and, more importantly, they are not close to normality. To substantiate this claim, a statistical description of RV residuals is given in Table 6, from which one can notice a clear departure from normality.

Table 4

Sample Description of the Realised Volatility

\begin{tabular}{|c|c|c|c|c|}
\hline & RVEU/JPY & RVEUR/USD & RVGBP/USD & RVUSD/CHF \\
\hline Mean & 4.1235 & 1.6485 & 1.1999 & 4.5388 \\
\hline Median & 2.5589 & 1.6192 & 0.7523 & 4.3668 \\
\hline Std. Dev. & 2.4636 & 0.2164 & 1.0883 & 1.1326 \\
\hline Skewness & 0.6304 & 0.4156 & 1.9691 & 0.2415 \\
\hline Kurtosis & 1.6333 & 2.5644 & 4.9427 & 2.1198 \\
\hline Jarque-Bera & 12.10095 & 3.081881 & 67.49416 & 3.528255 \\
\hline
\end{tabular}

Table 5

RV-ARMA Parameters

\begin{tabular}{|c|c|c|c|c|c|c|c|c|}
\hline \multicolumn{2}{|c|}{ GARCH/ARMA models } & $\alpha 1$ & $\alpha 2$ & $\alpha 3$ & $\beta_{1}$ & $\beta 2$ & $\beta 3$ & $R^{2}$ \\
\hline \multirow[t]{2}{*}{ RVEUR/USD } & Coeff & 0.87591 & & & 0.262 & & & 0.8467 \\
\hline & St. error & 0.05499 & & & 0.112 & & & \\
\hline \multirow[t]{2}{*}{ RVEUR/JPY } & Coeff & 0.97012 & & & & & & 0.8635 \\
\hline & St. error & 0.01079 & & & & & & \\
\hline \multirow[t]{2}{*}{ RVUSD/CHF } & Coeff. & 0.96525 & & & & & & 0.9242 \\
\hline & St. error & 0.03016 & & & & & & \\
\hline \multirow[t]{2}{*}{ RVGBP/USD } & Coeff. & 0.98309 & & & 0.753 & & & 0.8684 \\
\hline & St. error & 0.03540 & & & 0.075 & & & \\
\hline
\end{tabular}

Table 6

Realised Volatility—Residual Sample Description

\begin{tabular}{lcccc}
\hline & RESRVEUR/JPY & RESRVEUR/USD & RESRVGBP/USD & RESRVUSD/CHF \\
\hline Mean & 0.0252 & -0.0008 & 0.0028 & 0.0006 \\
Median & -0.0259 & -0.0030 & -0.0245 & -0.0014 \\
Maximum & 4.3297 & 0.2997 & 1.4482 & 1.6542 \\
Minimum & -0.6288 & -0.3566 & -0.3928 & -1.6458 \\
Std. Dev. & 0.4967 & 0.0848 & 0.1949 & 0.3183 \\
Skewness & 7.9633 & -0.3635 & 5.1396 & -0.3032 \\
Kurtosis & 69.6834 & 7.7022 & 38.4516 & 19.5623 \\
Jarque-Bera & $16,255.3100$ & 78.2944 & $4,711.8880$ & 949.9314 \\
\hline
\end{tabular}

On the other hand, the fact that RV residuals are not independent is revealed in the autocorrelation functions (AC) of the residuals, which are presented in Figure 1.

The results in Tables 3 and 6 further imply that neither of the methods, based on the second order moments, produces Gaussian residuals. Thus, high kurtosis and skewness demonstrate existence of the 
information contained in the centred higher order cumulants. However, this article tests the hypothesis that the higher order cumulant function (HOCF) persists to be different from zero, and therefore contains significant information in the GARCH forecasting errors, which has not been extracted by the standard parameter estimation method.

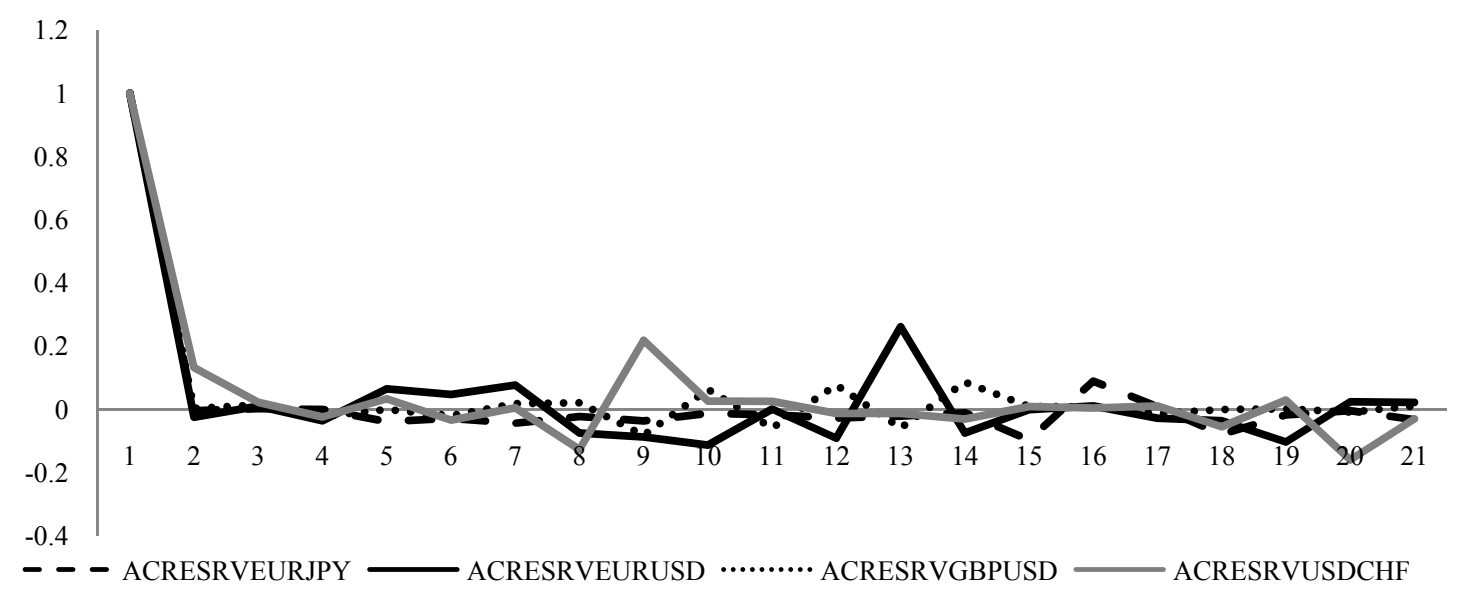

Figure 1. Realised volatility—Residual autocorrelation functions.

\section{GARCH and RV Modelling Using Higher Order Cumulants}

The leptokurtosis in the financial data is attributed to both volatility clustering and conditional non-normality (Bai, Russell, \& Tiao, 2001). A less restrictive GARCH model in which innovations are allowed to be non-normally distributed is introduced. It is shown that their GARCH specification can generate the excess kurtosis observed in most of the financial data and state that for heavy-tailed errors, the asymptotic distributions of quasi-maximum likelihood parameter estimators in ARCH and GARCH models are non-normal, and are particularly difficult to estimate using standard parametric methods.

In this article the following system of equations is used to calculate the third and the fourth cumulants:

$$
\begin{aligned}
\mathrm{C}_{x}^{3}(\tau 1, \tau 2) & =\left(\sum\left(x(t) \times\left(t+\tau_{1}\right) \times\left(t+\tau_{2}\right)\right) / n\right. \\
C_{x}^{4}(\tau 1, \tau 2, \tau 3) & =\left(\sum\left(x(t) \times\left(t+\tau_{1}\right) \times\left(t+\tau_{2}\right) \times\left(t+\tau_{3}\right)\right) / n\right.
\end{aligned}
$$

where $n$ is a number of observations and where the second-order cumulant $\mathrm{C}^{2}{ }_{x}(\tau)$ is just the autocorrelation function of the time series $x_{t}$. The zero lag cumulant of order $3 C_{x}^{3}(0,0)$ normalized by $\sigma_{x}^{3}$ has the skewness $\gamma_{x}^{3}$; $\mathrm{C}_{x}^{4}(0,0,0)$ normalized by $\sigma_{x}^{4}$ is known as kurtosis $\gamma_{x}^{4}$. An example of the third and fourth order cumulant functions is presented in Figure 2.

It is well known that heteroskedasticity of returns, $r_{t}$, implies (even more) heteroskedasticity in the squared returns $r_{t}^{2}$. So, parameters are estimated inefficiently and the usual standard errors are misleading. This article proposes the use of the HOC-based ARMA parameter estimation, which was suggested as a method of digital signal processing (Giannakis \& Delopoulos, 1995), but remained almost dormant for three decades.

The efficient AR parameter estimates can be obtained by solving the system of modified Yule Walker difference equation:

$$
\sum_{1}^{p} \alpha i C^{4}(k-i, k-1, k-m)=-C^{4}(k, k-1, k-m) \quad k \geq 1 \geq m \geq q+1
$$

The efficient MA parameter estimation can be performed by applying one of the algorithms, proposed by Swami; Q-slice algorithm (Swami \& Mendel, 1989), for instance. Q-slice algorithm uses autoregressive residuals calculated after estimating the AR parameters from equation (9). Following up, the impulse response 
parameters $I$ of the pure MA model of $x_{t}$ model are then estimated using cumulants:

$$
\begin{array}{cc}
x_{t}=\sum_{1}^{\infty} \beta_{j} v_{t-j} & i=1,2 \ldots \infty \\
\beta_{j}=\left(\sum_{1}^{p} \alpha i \mathrm{C}^{3}(q-i, j)\right) /\left(\sum_{1}^{p} \alpha i \mathrm{C}^{3}(q-i, 0)\right) & j=1,2 \ldots q
\end{array}
$$

After applying the HOC-GARCH parameter estimation, residuals are calculated and analyzed in terms of the third and the fourth order cumulants. The fourth order cumulant function is presented in Figure 3.

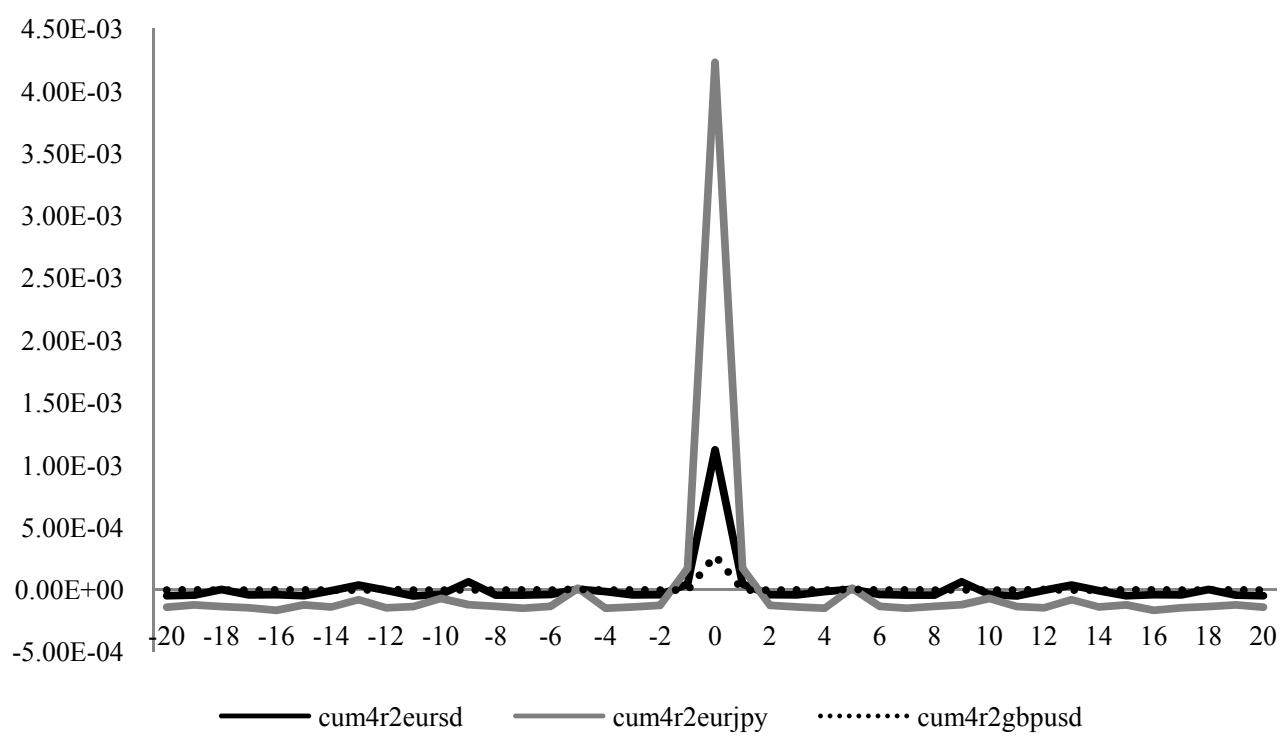

Figure 2. Fourth order cumulant function of EUR/USD squared returns.

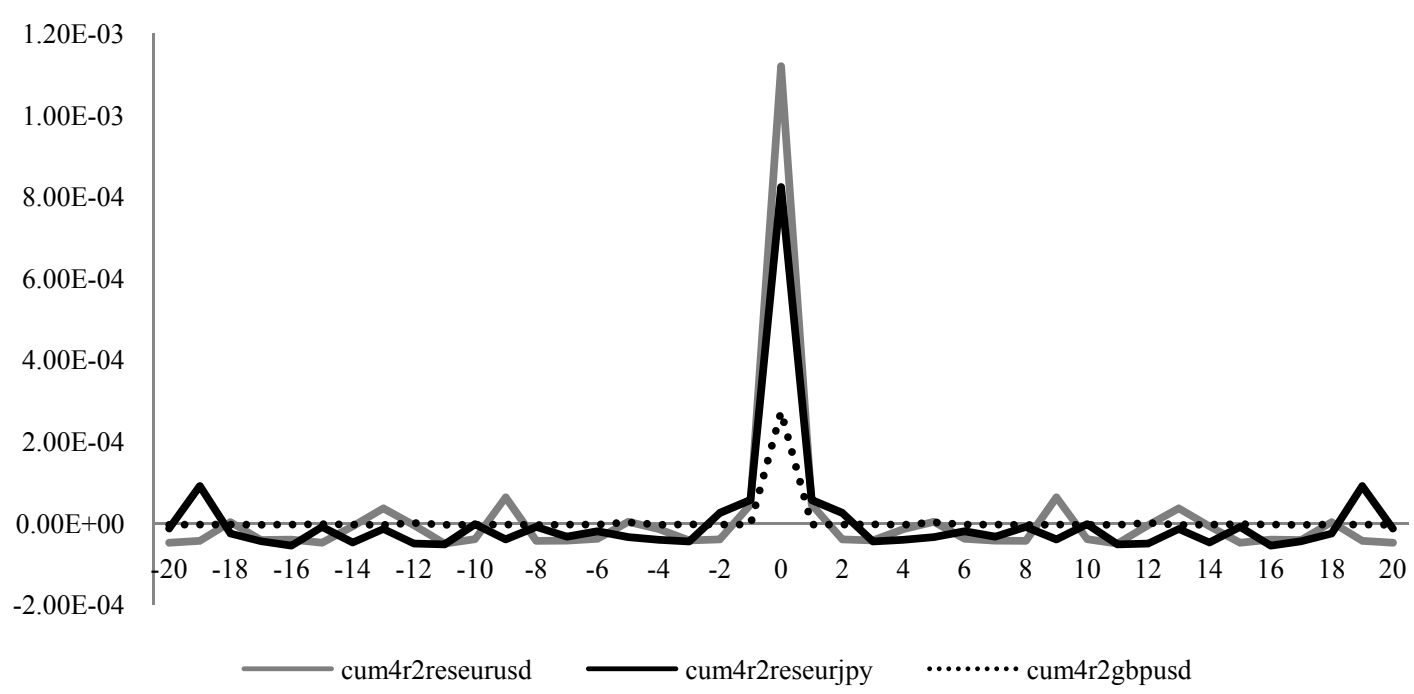

Figure 3. Fourth order cumulant function for FX model residuals.

The analysis of residual third and fourth order cumulants of different lags presented in Figures 2, 3, and 4 demonstrates that the HOC-GARCH estimation method is better at capturing empirical regularities found in the squared exchange rate returns and leaves residuals nearly independent.

Similar results are obtained using RV volatility. An example of the fourth order cumulant minimization is presented in Figure 5. 

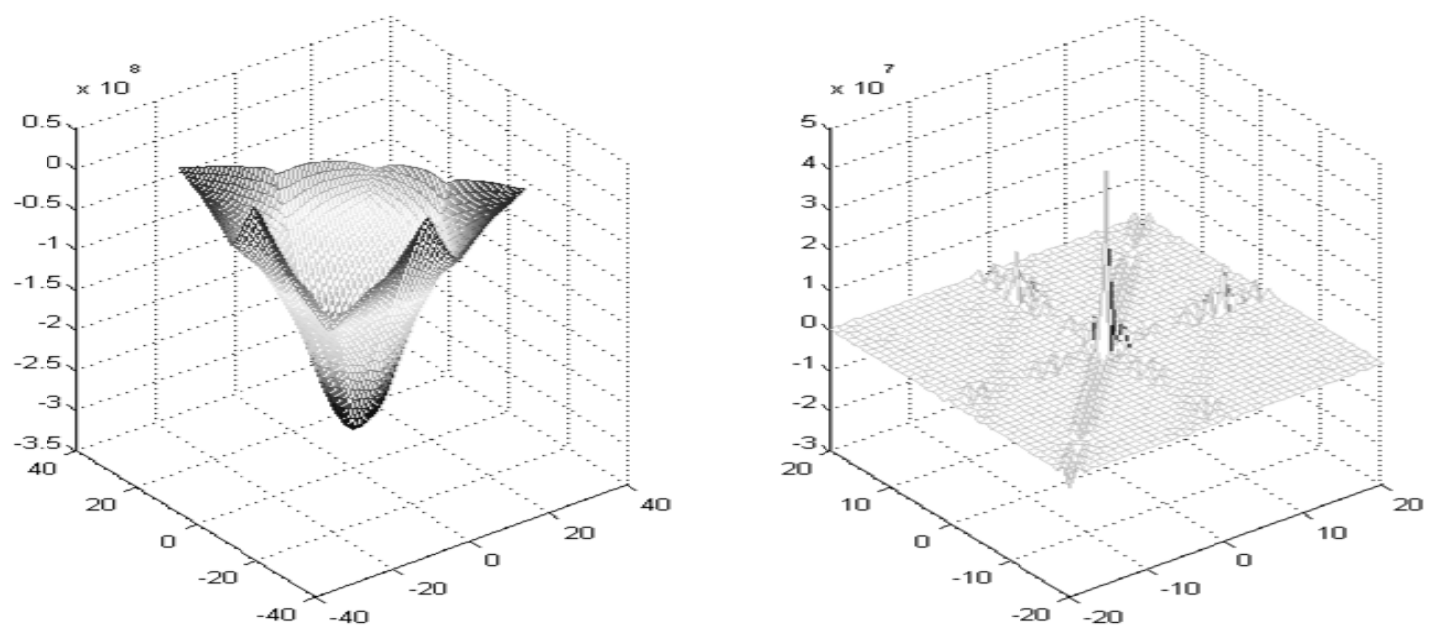

Figure 4. Fourth order cumulants of USD/CHF squared returns and HOC-GARCH residuals.
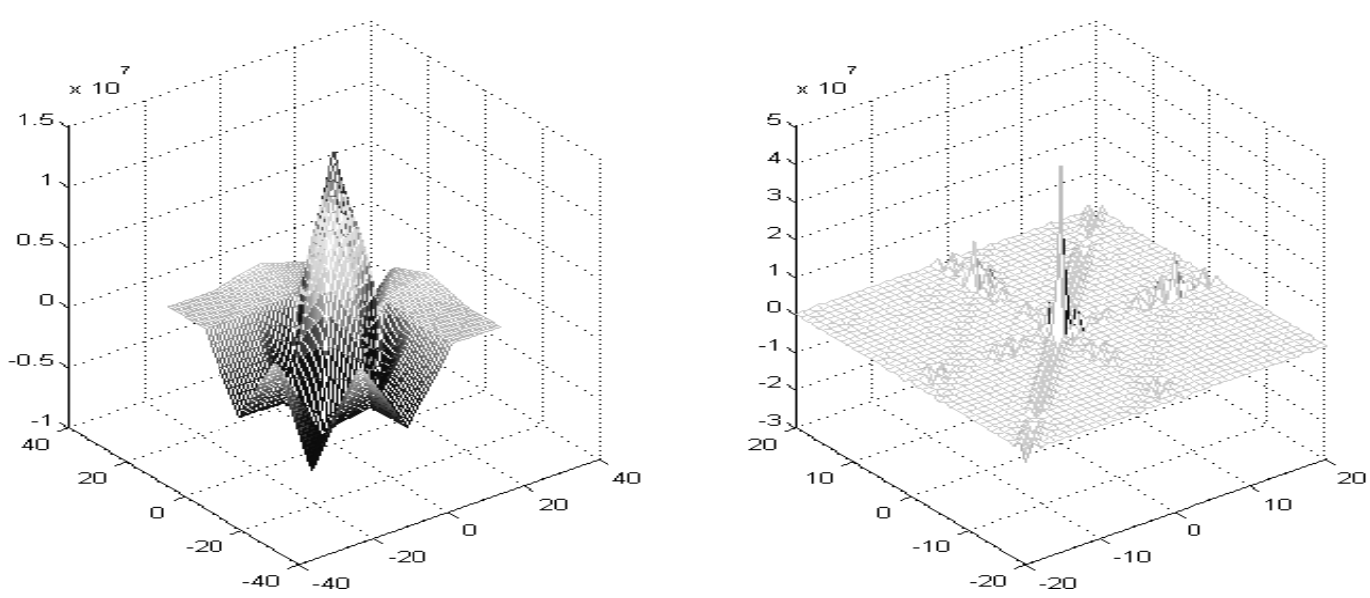

Figure 5. Fourth order cumulants: Realized volatility of GBPUSD and HOC residuals.

\section{Conclusions}

It has been demonstrated throughout this empirical analysis that GARCH-type models do not explain more than $35 \%$ of the exchange rate volatility, a purpose which is better served through the use of the HOC-based ARMA/GARCH parameter estimation method. The realised volatility model explains more than $80 \%$ of the exchange rate variance. However RV model parameters are close to the unit circle and therefore possibly stationary. GARCH and RV residuals have revealed that they, in fact, do not capture all the necessary information to forecast volatility. Thus, the paper introduces the ARMA parameter estimation method based on third and fourth order cumulants in order to capture more information about empirical regularities of FX squared returns. It is shown that superior whitening and independence of residuals is achieved if HOC ARMA parameter estimation is used. Further academic research studies in this area should focus on the problem of finding a sufficient statistic for ARMA parameter estimation, which goes beyond the covariance function, skewness, and kurtosis of the exchange rate volatility. 


\section{References}

Andersen, T. G., Bollerslev, T., Diebold, F. X., \& Ebens, H. (2001). The distribution of stock return volatility. Journal of Financial Economics, 61, 43-76.

Andersen, T. G., Bollerslev, T., Diebold, F. X., \& Labys, P. (2001). The distribution of exchange rate volatility. Journal of the American Statistical Association, 96, 42-55.

Andersen, T. G., Bollerslev, T., Diebold, F. X., \& Labys, P. (2003). Modeling and forecasting realized volatility. Econometrica, 71, 579-625.

Andreou, E., Pittis, N., \& Spanos, A. (2001). On modeling speculative process: The empirical literature. Journal of Economic Survey, 15(2), 187-220.

Bai, X., Russell, J. R., \& Tiao, G. C. (2003). Kurtosis of GARCH and stochastic volatility models with non-normality innovations. Journal of Econometrics, 114, 349-360.

Barndorff-Nielsen, O. E., \& Shepherd, N. (2002). Econometric analysis of realized volatility and its use in estimating stochastic volatility models. Journal of the Royal Statistical Society B, 64, 253-280.

Bollerslev, T. (1982). Generalized autoregressive conditional heteroskedasticity. In R. Engle (Ed.), ARCH selected readings (pp. 42-60). Oxford: Oxford UP.

Brooks, C. (1997). GARCH modeling in finance: A review of the software options. Economic Journal, 107, 1271-1276.

Carrol, R., \& Kearvey, C. (2009). GARCH modeling of stock market volatility. In G. Gregoriou (Ed.), Stock market volatility (pp. 71-90). London: Chapman Hall/CRC Press.

Cont, R. (2001). Empirical properties of asset returns: Stylized facts and statistical issues. Quantitative Finance, 1, $223-236$.

Corsi, A. (2009). Simple approximate long-memory model of realized volatility. Journal of Financial Econometrics, 7(2), 174-196.

Giannakis, B. G., \& Delopoulos, A. (1995). Cumulant-based autocorrelation estimation of non-Gaussian linear process. Signal Processing, 47, 1-17.

Giannakis, B. G., \& Mendel, M. M. (1990). Cumulant-based order determination of non-Gaussian acoustics. Speech and Signal Processing, 38(8), 1411-1423.

Mandelbrot, B. (1963). The variation of certain speculative prices. The Journal of Business, 36(4), 394-419.

Poon, H., \& Granger, C. (2003). Forecasting volatility in financial markets: A review. Journal of Economic Literature, 41, 478-539.

Porat, B., \& Friendlander, B. (1988). Performance analysis of parameter estimation algorithms based on higher order Moment. International Journal of Adaptive Control and Signal Processing, 3(3), 191-229.

Silvey, T. (2007). An investigation of the relative performance of GARCH models versus simple rules in forecasting volatility. In J. Knight, \& S. Satchel (Eds.), Forecasting volatility in the financial markets (pp. 101-130). Elsevier Finance, Quantitative Finance Series, Oxford.

Swami, A., \& Mendel, J. (1989). Closed form estimation of MA coefficients using autocorrelations and third-Order cumulants. Speech and Signal Processing, 37(11), 1794-1797. 